\title{
Generalised Weyl theorems and spectral pollution in the Galerkin method
}

\author{
Lyonell Boulton, Nabile Boussaïd, and Mathieu Lewin
}

\begin{abstract}
We consider a general framework for investigating spectral pollution in the Galerkin method. We show how this phenomenon is characterised via the existence of particular Weyl sequences which are singular in a suitable sense. For a semi-bounded selfadjoint operator $A$ we identify relative compactness conditions on a selfadjoint perturbation $B$ ensuring that the limiting set of spectral pollution of $A$ and $B$ coincide. Our results show that, under perturbation, this limiting set behaves in a similar fashion as the essential spectrum.
\end{abstract}

Mathematics Subject Classification (2010). Primary 35P15; Secondary 47J22.

Keywords. Weyl's Theorem, generalised essential spectrum, spectral pollution, Galerkin method.

\section{Introduction}

Let $A$ be a self-adjoint operator acting on a separable infinite dimensional Hilbert space $\mathscr{H}$ and let $\lambda$ be an isolated eigenvalue of $A$. For $\mathcal{I} \subset \mathbb{R}$ an interval let $\mathbb{1}_{\mathcal{I}}(A)$ be the spectral projector of $A$ associated to $\mathcal{I}$. The numerical estimation of $\lambda$ whenever $\inf \sigma_{\text {ess }}(A)<\lambda<\sup \sigma_{\text {ess }}(A)$ and, more generally, when

$$
\operatorname{Tr} \mathbb{1}_{(-\infty, \lambda)}(A)=\operatorname{Tr} \mathbb{1}_{(\lambda, \infty)}(A)=\infty,
$$

constitutes a serious challenge in applied spectral theory. Indeed, it is well established that classical approaches, such as the Galerkin method, suffer from variational collapse under no further restrictions on the approximating space. This often leads to numerical artefacts which do not belong to the spectrum of $A$, giving rise to what is generically called spectral pollution.

The spectral pollution phenomenon occurs in different practical contexts such as Sturm-Liouville operators (see [1], [32], and [31]), perturbations of periodic Schrödinger operators (see [7], and [23]) and systems underlying elliptic partial differential equations (see [2], [4], and [5]). It is a well documented difficulty in quantum chemistry and physics, in particular regarding relativistic computations; see 
[20], [30], [17], and [15]. It also plays a fundamental role in elasticity and magnetohydrodynamics; see [19], [10], [27], and [3]. In recent years this phenomenon has raised a large interest in the mathematical community; see [23], [18], [21], [12], [13], [26], and [25]. There are known pollution-free computational procedures alternative to the basic Galerkin method. These include specialised variational formulations such as those studied at length in [16], [19], [14], and [24] as well as general methods such as those proposed in [8], [6], [7], [21], and [12].

A natural approach to deal with spectral pollution is to derive conditions on the approximating subspaces guaranteeing a "safe" Galerkin method in a given interval of the real line. These conditions were found in [22] on an abstract setting for operators with particular block-type structures with respect to decompositions of the ambient Hilbert space. They turn out to be motivated from techniques in numerical analysis (see [2], [5], and [27]) and computational physics and chemistry (see references in [22]).

In the present paper we adopt a more general viewpoint than that of [22]. We establish an abstract framework for spectral pollution in the Galerkin method and then examine its invariance under relatively compact perturbations. Our main concern is primarily theoretical and general in nature. Nonetheless, however, we include various simple examples which illustrate the many subtleties faced when dealing with spectral pollution on a practical setting.

The technical context of our results can be summarised as follows. Let $\mathrm{D}(A)$ be the domain of $A$. Let $\mathscr{L}=\left(\mathscr{L}_{n}\right)_{n \in \mathbb{N}}$ be a sequence of finite dimensional subspaces of $\mathrm{D}(A)$, dense in the graph norm as $n \rightarrow \infty$ (Definition 1$)^{1}$. Denote by $A_{n}$ the compression of $A$ to $\mathscr{L}_{n}$. Denote by $\sigma(A, \mathscr{L})$ the large $n$ limiting set in Hausdorff distance of the Galerkin method spectra $\sigma\left(A_{n}\right)$, (Definition 2). Then $\sigma(A) \subset \sigma(A, \mathscr{L})$ (Proposition 2), however in general equality fails to occur in this identity. An abstract notion of limiting spectral pollution set can be formulated naturally as,

$$
\sigma_{\text {poll }}(A, \mathscr{L})=\sigma(A, \mathscr{L}) \backslash \sigma(A) .
$$

As it turns, points in the limiting spectral pollution set behave in a similar fashion as points in the essential spectrum (Proposition 3). Therefore a question arises: what sort of conditions on a perturbation $B$ ensure $\sigma_{\text {poll }}(A, \mathscr{L})=\sigma_{\text {poll }}(B, \mathscr{L})$ ? Below we establish a theoretical framework in order to address this question.

Section 2 and 3 are devoted to a characterisation of $\sigma(A, \mathscr{L})$ in terms of special Weyl-type sequences ( $\mathscr{L}$-Weyl sequences) and its structural properties. In Definition 3 we consider a decomposition of $\sigma(A, \mathscr{L})$ as the union of a limiting essential spectrum associated with $\mathscr{L}, \sigma_{\mathrm{ess}}(A, \mathscr{L})$, and its limiting discrete spectrum counterpart, $\sigma_{\text {disc }}(A, \mathscr{L})$. The former contains both the true essential spectrum $\sigma_{\text {ess }}(A)$ and $\sigma_{\text {poll }}(A, \mathscr{L})$ (Proposition 5).

\footnotetext{
${ }^{1}$ Below we will often consider a slightly more general framework which covers important applications such as those involving the finite element method. In this framework we will only require that the subspaces $\mathscr{L}_{n}$ lie in the domain of the quadratic form associated to $A$ and that the sequence $\mathscr{L}$ is dense in the form sense. However, in this more general setting we restrict our attention to $A$ being semi-bounded.
} 
The purpose of sections 4 and 5 is to find conditions on $B$ ensuring

$$
\sigma_{\text {ess }}(B, \mathscr{L})=\sigma_{\text {ess }}(A, \mathscr{L}) .
$$

According to our main result (Theorem 11), when $A$ and $B$ are bounded from below and

$$
(A-a)^{1 / 2}(B-a)^{-1 / 2}-1
$$

is a compact operator for some $a$ negative enough, (1) holds true. Therefore, an approximating sequence $\mathscr{L}$ will not asymptotically pollute for $A$ in a given interval if and only if it does not pollute for $B$ in the same interval. This generalises [22], Corollary 2.5.

Our present approach consists in adapting to the context of limiting spectra, several classical results for the spectrum and essential spectrum. In turns, this leads to many unexpected difficulties which we will illustrate on a variety of simple examples. In particular, we establish (Theorem 7) a limiting spectra version of the spectral mapping theorem allowing to replace the unbounded operator $A$ by its (bounded) resolvent $(A-a)^{-1}$. Remarkably, this theorem fails in general (Remark 4) for operators which are not semi-bounded.

Acknowledgement. The authors would like to acknowledge financial support from the British-French project PHC Alliance no. 22817YA. Partial support from the French Ministry of Research (ANR-10-BLAN-0101) is also acknowledged. They would also like to thank Éric Ricard for useful discussions.

\section{Limiting spectra}

We will often restrict our attention to $A$ being bounded from below, however we do not require this for the moment. Unless otherwise specified, we always assume that the subspaces $\mathscr{L}_{n}$ are dense in the following precise sense.

Definition 1 ( $A$-regular Galerkin sequences). We say that $\mathscr{L}=\left(\mathscr{L}_{n}\right), \mathscr{L}_{n} \subset \mathrm{D}(A)$, is an $A$-regular Galerkin sequence, or simply an $A$-regular sequence, if for all $f \in \mathrm{D}(A)$ there exists a sequence of vectors $\left(f_{n}\right)$ with $f_{n} \in \mathscr{L}_{n}$ such that $f_{n} \rightarrow f$ in the graph norm of $A$, that is

$$
\left\|f_{n}-f\right\|+\left\|A f_{n}-A f\right\| \stackrel{n \rightarrow \infty}{\longrightarrow} 0 .
$$

The orthogonal projection in the scalar product of $\mathscr{H}$ onto $\mathscr{L}_{n}$ will be denoted by $\pi_{n}: \mathscr{H} \rightarrow \mathscr{L}_{n}$ and the compression of $A$ to $\mathscr{L}_{n}$ by $A_{n}=\pi_{n} A \nmid \mathscr{L}_{n}: \mathscr{L}_{n} \rightarrow \mathscr{L}_{n}$. These compressions will sometimes be identified with any of their matrix representations. On sequences $\left(x_{n}\right)_{n \in \mathbb{N}} \subset \mathscr{H}$ of vectors and $\left(\mathscr{L}_{n}\right)_{n \in \mathbb{N}}$ of subspaces $\mathscr{L}_{n} \subset \mathrm{D}(A)$ we will often suppress the index and write $\left(x_{n}\right)$ and $\left(\mathscr{L}_{n}\right)$ instead. We will denote by 
$x_{n} \rightarrow x$ the fact that $x_{n}$ is weakly convergent to $x \in \mathscr{H}$. When the norm is not specified, $x_{n} \rightarrow x$ will denote the fact that $\left\|x_{n}-x\right\| \rightarrow 0$.

When $A$ is semi-bounded, we may also consider sequences $\mathscr{L}=\left(\mathscr{L}_{n}\right)$ only in the form domain of $A$. They may approximate the latter but not necessarily the operator domain. If $A \geq 0$, for instance, this simply means that $\mathscr{L}_{n} \subset \mathrm{D}\left(A^{1 / 2}\right)$ and $\mathscr{L}$ is $A^{1 / 2}$-regular but not necessarily $A$-regular. In our notation, for $x_{n} \in \mathscr{L}_{n}$, $A x_{n} \in \mathrm{D}\left(A^{1 / 2}\right)^{\#}$, the dual of $\mathrm{D}\left(A^{1 / 2}\right)$ as subspace of $\mathscr{H}$. Since $\pi_{n} A^{1 / 2} y \in$ $\mathscr{H}^{\#}=\mathscr{H}$ and $\pi_{n} A^{1 / 2} y \perp g$ for any $y \in \mathscr{H}$ and $g \in \mathscr{H} \ominus \mathscr{L}_{n}$, the compression $\pi_{n} A \uparrow \mathscr{L}_{n}: \mathscr{L}_{n} \rightarrow \mathscr{L}_{n}$ is well defined also in this framework. Moreover, a matrix representation of $A_{n}$ can be obtained in the usual manner, via $\left[\left\langle A^{1 / 2} b_{j}, A^{1 / 2} b_{k}\right\rangle\right]_{j k=1}^{\operatorname{dim} \mathscr{L}_{n}}$ for a given orthonormal basis $\left\{b_{j}\right\}$ of $\mathscr{L}_{n}$. As we think that there is no possible confusion, we will also denote the duality product associated to $w \in \mathrm{D}\left(A^{1 / 2}\right)^{\#}$ by $z \longmapsto\langle z, w\rangle$.

When $A$ is not semi-bounded but its essential spectrum has a gap containing a number $a$, we could as well consider sequences $\left(\mathscr{L}_{n}\right)$ which are only $|A-a|^{1 / 2}$-regular. We have chosen to avoid mentioning quadratic forms for operators which are not semi-bounded, because in practical applications (such as those involving the Dirac operator) the domain of $|A-a|^{1 / 2}$ does not necessarily coincide with the natural domain upon which the quadratic form is defined.

The limiting spectrum of $A$ relative to the Galerkin sequence $\mathscr{L}$, is the set of all limit points, up to subsequences, of the spectra of $A_{n}$ in the large $n$ limit.

Definition 2 (Limiting spectrum). The limiting spectrum of $A$ relative to $\mathscr{L}, \sigma(A, \mathscr{L})$, is the set of all $\lambda \in \mathbb{R}$ for which there exists $\lambda_{k} \in \sigma\left(A_{n_{k}}\right)$ such that $n_{k} \rightarrow \infty$ and $\lambda_{k} \rightarrow \lambda$ as $k \rightarrow \infty$.

Since all $A_{n}$ are Hermitian endomorphisms, $\sigma(A, \mathscr{L}) \subset \mathbb{R}$. The following lemma provides an alternative characterisation of $\sigma(A, \mathscr{L})$.

Lemma 1 (L्L-Weyl sequences). The real number $\lambda \in \sigma(A, \mathscr{L})$ if and only if there exists a sequence $x_{k} \in \mathscr{L}_{n_{k}}$ such that $\left\|x_{k}\right\|=1$ and $\pi_{n_{k}}(A-\lambda) x_{k} \rightarrow 0$ as $k \rightarrow \infty$.

Proof. According to the definition, $\lambda \in \sigma(A, \mathscr{L})$ if and only if there exists $\lambda_{k} \in \mathbb{R}$ and $x_{k} \in \mathscr{L}_{n_{k}}$ with $\left\|x_{k}\right\|=1$ such that $\lambda_{k} \rightarrow \lambda$ and $\pi_{n_{k}}\left(A-\lambda_{k}\right) x_{k}=0$. As $\pi_{n_{k}}(A-\lambda) x_{k}=\left(\lambda_{k}-\lambda\right) x_{k} \rightarrow 0$, one implication follows immediately.

On the other hand, let $\left(x_{k}\right)$ be as stated. Since the $A_{n}$ are Hermitian, there necessarily exists $\lambda_{k} \in \sigma\left(A_{n_{k}}\right)$ such that $\left|\lambda_{k}-\lambda\right| \leq\left\|\left(A_{n_{k}}-\lambda\right) x_{k}\right\| \rightarrow 0$. Thus $\lambda \in \sigma(A, \mathscr{L})$ ensuring the complementary implication.

We call $\left(x_{k}\right)$ an $\mathscr{L}$-Weyl sequence for $\lambda \in \sigma(A, \mathscr{L})$, by analogy to the classical notion of Weyl sequence [11].

Remark 1. Selfadjointness of $A_{n}$ is crucial in Lemma 1 . We illustrate this by means of a simple example. Let $\mathscr{H}=\ell^{2}(\mathbb{N})$ and $\left(e_{j}\right) \subset \mathscr{H}$ be the canonical orthonormal basis 
of this space. Let $A$ be the left shift operator defined by the condition $A: e_{j} \mapsto e_{j-1}$ with the convention $e_{0}=0$. Let $\mathscr{L}_{k}=\operatorname{Span}\left\{e_{i}, i \leq k\right\}$. For this data an analogous of Lemma 1 is no longer valid. Indeed, if $|\lambda|<1$ and

$$
x_{k} \stackrel{\text { def }}{=} \sqrt{\frac{1-|\lambda|^{2}}{1-|\lambda|^{2 k}}} \sum_{i=1}^{k} \lambda^{i-1} e_{i},
$$

then $x_{k} \in \mathscr{L}_{k},\left\|x_{k}\right\|=1$ and

$$
\left\|A x_{k}-\lambda x_{k}\right\|=\sqrt{\frac{1-|\lambda|^{2}}{1-|\lambda|^{2 k}}}|\lambda|^{k} \longrightarrow 0 .
$$

Therefore any point of the open unit disk is associated with an $\mathscr{L}$-Weyl sequence. On the other hand, however, $A_{n}$ is a Jordan block, so $\sigma\left(A_{n}\right)=\{0\}$ for all $n \in \mathbb{N}$ and hence necessarily $\sigma(A, \mathscr{L})=\{0\}$.

The above characterisation of points in the limiting spectrum combined with the minimax principle yields the following fundamental statement.

Proposition 2 (The limiting spectrum and the spectrum). Let $\mathscr{L}$ be an A-regular Galerkin sequence or, if $A \geq 0$, an $A^{1 / 2}$-regular Galerkin sequence. Then,

$$
\sigma(A) \subset \sigma(A, \mathscr{L})
$$

and

$$
\sigma_{\text {poll }}(A, \mathscr{L}) \stackrel{\text { def }}{=} \sigma(A, \mathscr{L}) \backslash \sigma(A) \subset\left(\ell^{-}, \ell^{+}\right)
$$

where

$$
\ell^{-} \stackrel{d e f}{=} \begin{cases}-\infty & \text { for } \inf \sigma(A)=-\infty \\ \inf \sigma_{\mathrm{ess}}(A) & \text { otherwise }\end{cases}
$$

and

$$
\ell^{+} \stackrel{\text { def }}{=} \begin{cases}+\infty & \text { for } \sup \sigma(A)=+\infty \\ \sup \sigma_{\mathrm{ess}}(A) & \text { otherwise }\end{cases}
$$

Proof. We start with the general case of an $A$-regular sequence. The classical characterisation of the spectrum of selfadjoint operators ensures that $\lambda \in \sigma(A)$ if and only if there is a normalised sequence $\left(y_{k}\right) \subset \mathrm{D}(A)$ such that $\left\|(A-\lambda) y_{k}\right\| \rightarrow 0$ (that is $\left(y_{k}\right)$ is a Weyl sequence for $\lambda$ ). We will now construct an $\mathscr{L}$-Weyl sequence from $\left(y_{k}\right)$. According to (3), we can find $\left(x_{m}^{k}\right)_{(k, m) \in \mathbb{N}^{2}}$ such that $x_{m}^{k} \in \mathscr{L}_{m},\left(y_{k}-x_{m}^{k}\right) \rightarrow 0$ and $(A-\lambda)\left(y_{k}-x_{m}^{k}\right) \rightarrow 0$ as $m \rightarrow \infty$. By virtue of a diagonal process, we can extract 
a subsequence such that $\pi_{m_{k}}(A-\lambda) x_{m_{k}}^{k} \rightarrow 0$. Dividing by $\left\|x_{m_{k}}^{k}\right\|$ (which does not vanish in the $k \rightarrow \infty$ limit), gives (4) as consequence of Lemma 1.

When $A \geq 0$ and $\mathscr{L}$ is only $A^{1 / 2}$-regular, a similar proof applies. We explicitly take $x_{m}^{k} \stackrel{\text { def }}{=} \pi_{m}^{\prime} y_{k}$ where $\pi_{m}^{\prime}$ is the orthogonal projection onto $\mathscr{L}_{m}$, for the scalar product furnished by the quadratic form associated to $A$. For all $z \in \mathscr{L}_{m}$ with $\|z\|=1$

$$
\begin{aligned}
\left|\left\langle z,(A-\lambda) x_{m}^{k}\right\rangle\right| & =\left|\left\langle z,(A-\lambda) y_{k}\right\rangle+(\lambda+1)\left\langle z, y_{k}-x_{m}^{k}\right\rangle\right| \\
& \leq\left\|(A-\lambda) y_{k}\right\|+(\lambda+1)\left\|y_{k}-x_{m}^{k}\right\|,
\end{aligned}
$$

where we have used that $\left\langle z,(A+1) y_{k}\right\rangle=\left\langle z,(A+1) \pi_{m}^{\prime} y_{k}\right\rangle$ by definition of the projection $\pi_{m}^{\prime}$. Thus

$$
\left\|\pi_{m}(A-\lambda) x_{m}^{k}\right\| \leq\left\|(A-\lambda) y_{k}\right\|+(\lambda+1)\left\|y_{k}-x_{m}^{k}\right\| .
$$

Our assumption that $\mathscr{L}$ is $A^{1 / 2}$-regular implies that $x_{m}^{k} \rightarrow y_{k}$ in $\mathrm{D}\left(A^{1 / 2}\right)$ when $m \rightarrow \infty$. Hence, the desired conclusion is achieved, once again, by a diagonal argument.

The proof of (5) is a classical consequence of the minimax principle. It may be found, for instance, in [21], Theorem 2.1, as well as [25] and [13].

In [22], Theorem 1.4, the existence of an $A$-regular Galerkin sequence $\mathscr{L}$ such that $\sigma(A, \mathscr{L})=\left[\ell^{-}, \ell^{+}\right]$is shown. Therefore the inclusion complementary to (4) does not hold in general. This is a source of difficulties in applications as there is no known systematic procedure able to identify $A$-regular Galerkin sequences such that $\sigma(A)=\sigma(A, \mathscr{L})$. By virtue of $(5)$, limiting spectral pollution $\sigma_{\text {poll }}(A, \mathscr{L})$ can only occur in "gaps" of the essential spectrum.

Let us now see how $\sigma_{\text {poll }}(A, \mathscr{L})$ can be characterised in a more precise manner in terms of particular $\mathscr{L}$-Weyl sequences.

Definition 3 (Limiting essential spectrum). We denote by $\sigma_{\text {ess }}(A, \mathscr{L})$ the set of all $\lambda \in \sigma(A, \mathscr{L})$ for which there exists an $\mathscr{L}$-Weyl sequence $\left(x_{k}\right)$ as in Lemma 1 with the additional property that $x_{k} \rightarrow 0$.

By analogy to the classical notions, we will call $\sigma_{\mathrm{ess}}(A, \mathscr{L})$ the limiting essential spectrum of $A$ associated to $\mathscr{L}$ and the corresponding sequence $\left(x_{k}\right)$ a singular $\mathscr{L}$ Weyl sequence.

Remark 2. From the definition it follows that $\sigma_{\text {ess }}(A+K, \mathscr{L})=\sigma_{\text {ess }}(A, \mathscr{L})$ for any selfadjoint operator $K \in \mathcal{K}(\mathscr{H})$.

Definition 4 (Limiting discrete spectrum). The residual set $\sigma_{\text {disc }}(A, \mathscr{L})=\sigma(A, \mathscr{L}) \backslash$ $\sigma_{\text {ess }}(A, \mathscr{L})$, will be called the limiting discrete spectrum of $A$ associated to $\mathscr{L}$. 
We illustrate these definitions by means of various simple examples.

Example 1 ( $A$ a bounded operator). Let $\mathscr{H}=\operatorname{Span}\left\{e_{n}^{ \pm}\right\}_{n \in \mathbb{N}}$ where $e_{n}^{ \pm}$is an orthonormal set of vectors in a given scalar product. Let $\mathscr{L}_{n}=\operatorname{Span}\left\{e_{1}^{ \pm}, \ldots, e_{n-1}^{ \pm}, f_{n}\right\}$ where $f_{n}=(\cos \theta) e_{n}^{+}+(\sin \theta) e_{n}^{-}$for $\theta \in(0, \pi / 2)$. Let $^{2}$

$$
A=\sum_{n \geq 1}\left|e_{n}^{+}\right\rangle\left\langle e_{n}^{+}\right|,
$$

that is, $A$ is the orthogonal projector onto $\operatorname{Span}\left(e_{n}^{+}\right)$and $\sigma(A)=\sigma_{\text {ess }}(A)=\{0,1\}$. Then $\sigma\left(A_{n}\right)=\left\{0,1, \cos ^{2} \theta\right\}$ for all $n$ and $\sigma(A, \mathscr{L})=\sigma_{\text {ess }}(A, \mathscr{L})=\left\{0,1, \cos ^{2} \theta\right\}$. Here $x_{n}=e_{n}^{-}$is a singular $\mathscr{L}$-Weyl sequence associated to $\lambda=0, x_{n}=e_{n}^{+}$is a singular $\mathscr{L}$-Weyl sequence associated to $\lambda=1$ and $x_{n}=f_{n}$ is a singular $\mathscr{L}$-Weyl sequence associated to $\lambda=\cos ^{2} \theta$.

Example 2 ( $A$ a semi-bounded operator). Let $\mathscr{H}$ be as in Example 1 and define

$$
\mathscr{L}_{n}=\operatorname{Span}\left\{e_{1}^{ \pm}, \ldots, e_{n-1}^{ \pm}, e_{n}^{-}\right\}
$$

For $f_{n}^{ \pm}=\sin \left(\frac{1}{n}\right) e_{n}^{\mp} \pm \cos \left(\frac{1}{n}\right) e_{n}^{ \pm}$, let

$$
A=\sum n^{2}\left|f_{n}^{+}\right\rangle\left\langle f_{n}^{+}\left|-\sum\right| f_{n}^{-}\right\rangle\left\langle f_{n}^{-}\right|
$$

which has a $2 \times 2$ block diagonal representation in the basis $\left(e_{n}^{ \pm}\right)$. Then $\sigma_{\text {ess }}(A)=$ $\{-1\}$ and $\sigma_{\text {disc }}(A)=\left\{n^{2}: n \in \mathbb{N}\right\}$. On the other hand

$$
\sigma\left(A_{n}\right)=\left\{-1, n^{2} \sin ^{2} \frac{1}{n}-\cos ^{2} \frac{1}{n}, 1, \ldots,(n-1)^{2}\right\},
$$

where -1 is an eigenvalue of multiplicity $n-1$. Therefore

$$
\sigma_{\text {ess }}(A, \mathscr{L})=\{-1,0\} \quad \text { and } \quad \sigma_{\text {disc }}(A, \mathscr{L})=\left\{n^{2}: n \in \mathbb{N}\right\} .
$$

The former is a consequence of Proposition 3(ii) while the latter follows from Proposition 5(iii) below.

We can verify directly the validity of the latter as follows. Assume that conversely $\left(x_{k}\right)$ was a singular $\mathscr{L}$-Weyl sequence associated with $v^{2} \in \sigma_{\text {disc }}(A)$. Then we have $\pi_{n_{k}}\left(A-v^{2}\right) x_{k} \rightarrow 0$ and $x_{k} \rightarrow 0$. For $m<n_{k}$

$$
p_{m} \pi_{n_{k}}\left(A-v^{2}\right) x_{k}=\left(A-v^{2}\right) p_{m} x_{k}
$$

where $p_{m}=\sum_{i \leq m}\left|f_{i}^{ \pm}\right\rangle\left\langle f_{i}^{ \pm}\right|$. Then, on the one hand,

$$
\left\|p_{n_{k}-1} x_{k}-\left\langle f_{v}^{+}, x_{k}\right\rangle f_{v}^{+}\right\|^{2} \leq\left\|\left(A-v^{2}\right) p_{n_{k}-1} x_{k}\right\|^{2} \longrightarrow 0
$$

\footnotetext{
${ }^{2}$ Here and elsewhere we use the bra-ket notation $|f\rangle\langle g|$ to denote the linear operator $\psi \mapsto\langle g, \psi\rangle f$.
} 
so that $\left\|p_{n_{k}-1} x_{k}\right\|^{2}+\left\|\left(A-v^{2}\right) p_{n_{k}-1} x_{k}\right\|^{2} \rightarrow 0$ as $k \rightarrow \infty$. On the other hand,

$$
\left(A-v^{2}\right)\left(x_{k}-\left\langle e_{n_{k}}^{-}, x_{k}\right\rangle e_{n_{k}}^{-}\right)=\left(A-v^{2}\right) p_{n_{k}-1} x_{k} .
$$

Since $\left|\left\langle\left(A-v^{2}\right) e_{n}^{-}, e_{n}^{-}\right\rangle\right|=\left|n^{2} \sin ^{2} \frac{1}{n}+\cos ^{2} \frac{1}{n}-v^{2}\right| \rightarrow\left|2-v^{2}\right|>0$, projecting each term onto $\mathscr{L}_{n_{k}}$ yields $\left\langle e_{n_{k}}^{-}, x_{k}\right\rangle \rightarrow 0$ also. But then $1=\left\|x_{k}\right\| \rightarrow 0$, which is a contradiction, so there are no singular $\mathscr{L}$-Weyl sequences for $v^{2}$.

Example 3 ( $A$ a strongly indefinite operator). Let $\mathscr{H}$ and $\mathscr{L}_{n}$ be as in Example 2. Let $f_{n}^{ \pm}=\frac{1}{\sqrt{2}} e_{n}^{+} \pm \frac{1}{\sqrt{2}} e_{n}^{-}$. Let

$$
A=\sum n\left|f_{n}^{+}\right\rangle\left\langle f_{n}^{+}\left|-\sum n\right| f_{n}^{-}\right\rangle\left\langle f_{n}^{-}\right| .
$$

Then $\sigma(A)=\{ \pm n: n \in \mathbb{N}\}=\sigma_{\text {disc }}(A)$. On the other hand

$$
\sigma(A, \mathscr{L})=\mathbb{Z}, \quad \sigma_{\text {ess }}(A, \mathscr{L})=\{0\} \quad \text { and } \quad \sigma_{\text {disc }}(A, \mathscr{L})=\{ \pm n: n \in \mathbb{N}\} .
$$

The proof of the latter is similar to that of the analogous property in Example 2.

\section{Limiting spectra and the behaviour of singular $\mathscr{L}$-Weyl sequences}

We now examine more closely various basic properties of the limiting spectra $\sigma(A, \mathscr{L})$, $\sigma_{\text {ess }}(A, \mathscr{L})$ and $\sigma_{\text {disc }}(A, \mathscr{L})$. These properties can be deduced via an analysis of the behaviour of different types of $\mathscr{L}$-Weyl sequences.

Proposition 3 (Limiting essential and discrete spectra and the spectrum). Let $\mathscr{L}$ be an $A$-regular Galerkin sequence, or, if $A \geq 0$, an $A^{1 / 2}$-regular Galerkin sequence. Then

(i) the limiting spectrum $\sigma(A, \mathscr{L})$ and the limiting essential spectrum $\sigma_{\mathrm{ess}}(A, \mathscr{L})$ are closed subsets of $\mathbb{R}$;

(ii) moreover $\sigma_{\text {ess }}(A) \subset \sigma_{\text {ess }}(A, \mathscr{L})$ and $\sigma_{\text {disc }}(A, \mathscr{L}) \subset \sigma_{\text {disc }}(A)$.

Proof. The proof of (i) involves a standard diagonal argument and it is left to the reader. For the second statement we need the following auxiliary result which will be used repeatedly below.

Lemma 4. A sequence $x_{k} \in \mathscr{L}_{n_{k}}$ is such that $\left\|x_{k}\right\|=1, x_{k} \rightarrow x$ and $\pi_{n_{k}}(A-\lambda) x_{k}$ $\rightarrow 0$, only when $x \in \operatorname{Ker}(A-\lambda)$.

Proof of Lemma 4. Suppose that $\left(x_{k}\right)$ satisfies the hypothesis with $\mathscr{L}$ an $A$-regular Galerkin sequence. Let $f \in \mathrm{D}(A)$ and $f_{n} \in \mathscr{L}_{n}$ such that $f_{n} \rightarrow f$ in the norm of $\mathrm{D}(A)$. Then $\left\langle\pi_{n_{k}}(A-\lambda) x_{k}, f_{n_{k}}\right\rangle \rightarrow 0$. On the other hand, since $f_{k} \rightarrow f$ in $\mathrm{D}(A)$,

$$
\left\langle\pi_{n_{k}}(A-\lambda) x_{k}, f_{n_{k}}\right\rangle=\left\langle x_{k},(A-\lambda) f_{n_{k}}\right\rangle \rightarrow\langle x,(A-\lambda) f\rangle .
$$


Thus $\langle x,(A-\lambda) f\rangle=0$ for all $f \in \mathrm{D}(A)$, so that $x \in \mathrm{D}\left(A^{*}\right)=\mathrm{D}(A)$ and $(A-\lambda) x=0$ as required.

Suppose now that $A \geq 0$ and $\mathscr{L}$ is only $A^{1 / 2}$-regular. The hypothesis implies that $\left(x_{k}\right)$ is a bounded sequence in $\mathrm{D}\left(A^{1 / 2}\right)$. Then the proof reduces to the same argument, but taking this time $f_{n_{k}}$ in $\mathrm{D}\left(A^{1 / 2}\right)$.

We now turn to the proof of (ii) in Proposition 3. The fact that $\sigma_{\text {ess }}(A) \subset \sigma_{\text {ess }}(A, \mathscr{L})$ is proved similarly to (4). It should only be noted that the $\mathscr{L}$-Weyl sequence found for $\lambda \in \sigma_{\text {ess }}(A)$ additionally satisfies $x_{m_{k}}^{k} \rightarrow 0$. For the inclusion $\sigma_{\text {disc }}(A, \mathscr{L}) \subset$ $\sigma_{\text {disc }}(A)$ note that, if $\lambda \in \sigma_{\text {disc }}(A, \mathscr{L})$, there exists $x_{k} \in \mathscr{L}_{n_{k}}$ such that $\left\|x_{k}\right\|=1$, $x_{k} \rightarrow x \neq 0$ and $\pi_{n_{k}}(A-\lambda) x_{k} \rightarrow 0$. As $\lambda \notin \sigma_{\text {ess }}(A)$ (by the previous part), then either $\lambda \in \sigma_{\mathrm{disc}}(A)$ or $\lambda \notin \sigma(A)$. By Lemma 4 , the latter is impossible.

Remark 3. If $\sigma_{\text {ess }}(A)=\sigma_{\text {ess }}(A, \mathscr{L})$ then automatically $\sigma_{\text {disc }}(A)=\sigma_{\text {disc }}(A, \mathscr{L})$ and $\sigma(A)=\sigma(A, \mathscr{L})$.

We will now examine more closely singular $\mathscr{L}$-Weyl sequences associated to points $\lambda \in \sigma_{\mathrm{ess}}(A, \mathscr{L})$.

Proposition 5 (Singular $\mathscr{L}$-Weyl sequences). Let $\mathscr{L}$ be an A-regular Galerkin sequence, or, if $A \geq 0$, an $A^{1 / 2}$-regular Galerkin sequence. The real number $\lambda \in$ $\sigma_{\mathrm{ess}}(A, \mathscr{L})$ if and only if

(i) either $\lambda \notin \sigma(A)$ and there exists $\lambda_{k} \rightarrow \lambda$ and $y_{k} \in \mathscr{L}_{n_{k}}$ such that $y_{k} \rightarrow 0$ and $\pi_{n_{k}}\left(A-\lambda_{k}\right) y_{k}=0$;

(ii) or $\lambda \in \sigma_{\text {ess }}(A)$ and there exists $\lambda_{k} \rightarrow \lambda$ and $y_{k} \in \mathscr{L}_{n_{k}}$ such that $y_{k} \rightarrow 0$ and $\pi_{n_{k}}\left(A-\lambda_{k}\right) y_{k}=0$;

(iii) or $\lambda \in \sigma_{\mathrm{disc}}(A)$ and for any $\varepsilon>0$

$$
\operatorname{Rank}\left(\mathbb{1}_{(\lambda-\varepsilon, \lambda+\varepsilon)}\left(A_{n}\right)\right) \geq \operatorname{Rank}\left(\mathbb{1}_{\{\lambda\}}(A)\right)+1
$$

for all $n$ large enough.

In cases (i) and (iii), $\lambda$ can in some sense be regarded as a point of spectral pollution for $A$ relative to $\mathscr{L}$. In case (iii), $\lambda \in \sigma(A)$, but the multiplicity of the approximating spectrum $\sigma\left(A_{n}\right)$ is too large for $n$ large, leading to the wrong spectral representation of $A$ in the limit $n \rightarrow \infty$. In our definition of the polluted spectrum $\sigma_{\text {poll }}(A, \mathscr{L})$ in (5), we have chosen to require that $\lambda \notin \sigma(A)$, following [22]. Any $\lambda \in \sigma(A)$ satisfying (iii) could also be considered as a spurious spectral point. However, in case (ii), the singular $\mathscr{L}$-Weyl sequence $\left(y_{k}\right)$ behaves like a classical singular Weyl sequence.

Only in cases (i) and (ii) the existence of a singular $\mathscr{L}$-Weyl sequence $\left(y_{k}\right)$ consisting of exact eigenvectors of $A_{n_{k}}$ such that $\pi_{n_{k}}\left(A-\lambda_{k}\right) y_{k}=0$ and $\lambda_{k} \rightarrow \lambda$ is guaranteed. In case (iii) it may occur that all the eigenvectors of $A_{n_{k}}$ whose 
corresponding eigenvalue converges to $\lambda$, converge weakly to a non-zero element of $\operatorname{Ker}(A-\lambda)$, and that only a linear combination of these eigenvectors converges weakly to zero. This can be illustrated by means of a simple example.

Example 4 (Spectral point satisfying Proposition 5(iii)). Let $\mathscr{H}=\operatorname{Span}\left\{e_{0}, e_{n}^{ \pm}\right\}$ where $e_{0}, e_{n}^{ \pm}$form an orthonormal basis. Let

$$
A=\sum_{n=1}^{\infty}\left|e_{n}^{+}\right\rangle\left\langle e_{n}^{+}\left|-\sum_{n=1}^{\infty}\right| e_{n}^{-}\right\rangle\left\langle e_{n}^{-}\right| .
$$

Then $\sigma(A)=\{-1,0,1\}$ and $\sigma_{\text {ess }}(A)=\{-1,1\}$. The eigenvalue 0 has multiplicity one and associated eigenvector $e_{0}$. Let

$$
\mathscr{L}_{n}=\operatorname{Span}\left\{e_{1}^{ \pm}, \ldots, e_{n-1}^{ \pm}, f_{n}^{ \pm}\right\},
$$

where

$$
f_{n}^{ \pm}=\frac{e_{0}+\alpha_{n}^{ \pm} e_{n}^{+}-\alpha_{n}^{\mp} e_{n}^{-}}{\sqrt{1+\left(\alpha_{n}^{ \pm}\right)^{2}+\left(\alpha_{n}^{\mp}\right)^{2}}}
$$

for

$$
\alpha_{n}^{ \pm}= \pm \sqrt{\frac{1 \pm \frac{1}{n^{2}}}{2\left(1 \mp \frac{1}{n^{2}}\right)}} .
$$

Then $\sigma(A, \mathscr{L})=\{0, \pm 1\}=\sigma_{\text {ess }}(A, \mathscr{L})$. In this case $A_{n}$ has two eigenvalues approaching zero in the large $n$ limit, with corresponding eigenvectors $f_{n}^{+}$and $f_{n}^{-}$. It is readily seen that $f_{n}^{ \pm} \rightarrow e_{0} / \sqrt{2}$ and so only the difference $f_{n}^{+}-f_{n}^{-}$tends weakly to zero.

Proof of Proposition 5. Let $\lambda \in \sigma_{\text {ess }}(A, \mathscr{L}) \subset \sigma(A, \mathscr{L})$. By definition of $\sigma(A, \mathscr{L})$ there exists a normalised sequence $\left(y_{k}\right)$ such that $\pi_{n_{k}}\left(A-\lambda_{k}\right) y_{k}=0$ and $\lambda_{k} \rightarrow \lambda$. The main question is whether one can ensure that $y_{k} \rightarrow 0$ weakly. Up to extraction of a subsequence, we may assume that $y_{k} \rightarrow y \in \operatorname{Ker}(A-\lambda)$ (by Lemma 4). If $\lambda \notin \sigma(A)$, then $\operatorname{Ker}(A-\lambda)=\{0\}$ and necessarily $y=0$, thus (i) follows.

For the proof of (ii) we require the following auxiliary result.

Lemma 6. Let $\mathcal{V} \subset \mathrm{D}(A)$ be a subspace of dimension $d>0$, with associated orthogonal projector $\pi \mathcal{v}$. Let $\varepsilon>0$ be such that

$$
\left\|\pi_{\mathcal{V}}(A-\lambda) x\right\| \leq \varepsilon\|x\| \quad \text { for all } x \in \mathcal{V} .
$$

There exists $N>0$ and a sequence of spaces $\mathfrak{W}_{n} \subset \mathscr{L}_{n}$ of dimension $d$, such that for all $n \geq N$

$$
\left\|\pi w_{n}(A-\lambda) y\right\| \leq 2 \varepsilon \sqrt{d}\|y\| \quad \text { for all } y \in \mathcal{W}_{n} .
$$


Proof of Lemma 6 . We firstly assume that $\mathscr{L}$ is $A$-regular. Let $\left(e_{j}\right)$ be a fixed orthonormal basis of $\mathcal{V}$. Then there exists $e_{j}^{n} \in \mathscr{L}_{n}$ such that $\left\|e_{j}^{n}-e_{j}\right\|_{\mathrm{D}(A)} \rightarrow 0$ when $n \rightarrow \infty$. The Gram matrix $G_{n} \stackrel{\text { def }}{=}\left(\left\langle e_{i}^{n}, e_{j}^{n}\right\rangle\right)_{1 \leq i, j \leq d}$ converges to the $d \times d$ identity matrix as $n \rightarrow \infty$, and therefore, for sufficiently large $n, \mathbb{W}_{n} \stackrel{\text { def }}{=} \operatorname{span}\left\{e_{j}^{n}, j=1, \ldots, d\right\}$ has dimension $d$. Now we define an orthonormal basis for $\mathcal{W}_{n}$ by

$$
f_{j}^{n} \stackrel{\text { def }}{=} \sum_{k=1}^{d}\left(G_{n}^{-1 / 2}\right)_{k j} e_{k}^{n} .
$$

Since $\left(G_{n}^{-1 / 2}\right)_{k j} \rightarrow \delta_{k j}$ and $e_{j}^{n} \rightarrow e_{j}$ in the graph norm, it is then clear that

$$
\left\|e_{j}-f_{j}^{n}\right\|_{\mathrm{D}(A)} \longrightarrow 0, \quad n \longrightarrow \infty .
$$

This shows in particular that $\left\|\left(\pi w_{n}-\pi v\right) A\right\| \rightarrow 0,\left\|\pi w_{n}-\pi v\right\| \rightarrow 0$ and hence that $\left\|\pi w_{n}(A-\lambda) f_{j}^{n}-\pi_{\mathcal{v}}(A-\lambda) e_{j}\right\| \rightarrow 0$. Let $N>0$ be such that

$$
\left\|\pi w_{n}(A-\lambda) f_{j}^{n}\right\| \leq 2 \varepsilon \text { for all } n \geq N, j=1, \ldots, d .
$$

For $y=\sum_{j=1}^{d} \hat{y}_{j} f_{j}^{n} \in W_{n}$, we get

$$
\left\|\pi w_{n}(A-\lambda) y\right\| \leq 2 \varepsilon \sum_{j=1}^{d}\left|\hat{y}_{j}\right| \leq 2 \varepsilon \sqrt{d}\|y\|,
$$

which ensures the desired property.

When $A \geq 0$ and $\mathscr{L}$ is only $A^{1 / 2}$-regular, the proof is the same, using convergence in $\mathrm{D}\left(A^{1 / 2}\right)$ and the fact that $\left\|\left(\pi w_{n}-\pi \mathcal{V}\right) A^{1 / 2}\right\| \rightarrow 0$

The proof of (i) in Proposition 5 is achieved as follows. Assume that $\lambda \in \sigma_{\text {ess }}(A)$. For all $d \in \mathbb{N}$ there exists a subspace $\mathcal{V}_{d} \subset \mathrm{D}(A)$, such that $\operatorname{dim} \mathcal{V}_{d}=d^{2}$ and

$$
\|(A-\lambda) y\| \leq \frac{1}{d^{2}}\|y\| \quad \text { for all } y \in \mathcal{V}_{d},
$$

see for instance [11], Lemma 4.1.4. According to Lemma 6 and an inductive argument, there is a sequence $\left(n_{d}\right) \subset \mathbb{N}$ and $d^{2}$-dimensional subspaces $\mathfrak{W}_{d} \subset \mathscr{L}_{n_{d}}$, such that

$$
\left\|\pi_{n_{d}}(A-\lambda) y\right\| \leq \frac{2}{d}\|y\| \quad \text { for all } y \in \mathcal{W}_{d} .
$$

This ensures that $A_{n_{d}}$ has at least $d^{2}$ eigenvalues in the interval $[\lambda-2 / d, \lambda+2 / d]$.

Let $\left(f_{j}^{n_{d}}\right)_{j=1}^{d^{2}} \subset \mathscr{L}_{n_{d}}$ be an orthonormal set of $d^{2}$ eigenvectors of $A_{n_{d}}$, with associated eigenvalues $\left(\lambda_{j}^{n_{d}}\right)_{j=1}^{d^{2}}$ satisfying $\left|\lambda_{j}^{n_{d}}-\lambda\right| \leq 2 / d$. We inductively define 
the following singular $\mathscr{L}$-Weyl sequence for $\lambda$ :

$$
\begin{aligned}
& y_{1}=f_{1}^{n_{1}}, \\
& y_{2}=f_{\delta_{2}}^{n_{2}} \quad \text { with } 1 \leq \delta_{2} \leq 2^{2} \text { such that }\left|\left\langle y_{2}, y_{1}\right\rangle\right| \leq 1 / \sqrt{2} \text {, } \\
& y_{3}=f_{\delta_{3}}^{n_{3}} \quad \text { with } 1 \leq \delta_{3} \leq 3^{2} \text { such that }\left|\left\langle y_{3}, y_{j}\right\rangle\right| \leq 1 / \sqrt{3} \text { for } j=1,2 \text {, } \\
& y_{d}=f_{\delta_{d}}^{n_{d}} \quad \text { with } 1 \leq \delta_{d} \leq d^{2} \text { such that } \\
& \left|\left\langle y_{d}, y_{j}\right\rangle\right| \leq 1 / \sqrt{d} \text { for } j=1, \ldots, d-1 .
\end{aligned}
$$

The existence of $\delta_{d}$ is guaranteed by the fact that

$$
1=\left\|y_{k}\right\|^{2} \geq \sum_{j=1}^{d^{2}}\left|\left\langle y_{k}, f_{j}^{n_{d}}\right\rangle\right|^{2} \quad \text { for all } k=1, \ldots, d-1 .
$$

Indeed, there are at most $d$ indices $j$ in the above summation, such that $\left|\left\langle y_{k}, f_{j}^{n_{d}}\right\rangle\right|^{2} \geq$ $1 / d$. Hence, in total, there are at most $d(d-1)$ indices $j$ such that $\left|\left\langle y_{k}, f_{j}^{n_{d}}\right\rangle\right|^{2} \geq$ $1 / d$ for at least one $k=1, \ldots, d-1$. Since $d(d-1)<d^{2}$ for $d \geq 1$, we deduce that there is at least one index $j \stackrel{\text { def }}{=} \delta_{d}$ such that $\left|\left\langle y_{k}, f_{j}^{n_{d}}\right\rangle\right|^{2} \leq 1 / d$ for all $k=1, \ldots, d-1$. By construction $\left\|y_{d}\right\|=1$ and $\left|\left\langle y_{i}, y_{j}\right\rangle\right| \leq 1 / \sqrt{\max (i, j)}$. Thus $y_{k} \rightarrow 0$ as $k \rightarrow \infty$, ensuring (ii).

Note that, conversely, if (i) or (ii) holds true, then $\lambda \in \sigma_{\text {ess }}(A, \mathscr{L})$ by Definition 3.

Let us now prove that if $\lambda \in \sigma_{\text {ess }}(A, \mathscr{L}) \cap \sigma_{\text {disc }}(A)$, then (iii) holds true. Let $x_{k} \in \mathscr{L}_{n_{k}}$ be a singular $\mathscr{L}$-Weyl sequence: $\pi_{n_{k}}(A-\lambda) x_{k} \rightarrow 0,\left\|x_{k}\right\|=1$ and $x_{k} \rightarrow 0$. Let $\mathcal{V}=\operatorname{Ker}(A-\lambda) \neq\{0\}$ and $d=\operatorname{dim}(\mathcal{V})$. For $n$ sufficiently large there is a space $\mathfrak{W}_{n} \subset \mathscr{L}_{n}$ of dimension $d$ such that for all $\varepsilon>0$, there exists $N>0$ such that

$$
\left\|\pi_{n}(A-\lambda) y\right\| \leq \varepsilon\|y\| \quad \text { for all } y \in W_{n}
$$

whenever $n \geq N$. Let $S_{k}=\operatorname{Span}\left\{W_{n_{k}}, x_{k}\right\}$. Since $x_{k} \rightarrow 0$ and $W_{n_{k}}$ does not increase in dimension in the large $k$ limit, necessarily $\operatorname{dim}\left(S_{k}\right)=d+1$ for all $k$ large enough. For all $\varepsilon>0$ there exists $M>0$ such that

$$
\left\|\pi_{n_{k}}(A-\lambda) y\right\| \leq \varepsilon\|y\| \quad \text { for all } y \in S_{k}
$$

whenever $k \geq M$. This ensures that $\sigma\left(A_{n_{k}}\right) \cap(\lambda-\varepsilon, \lambda+\varepsilon)$ contains at least $d+1$ points counting multiplicity and hence the claimed conclusion is achieved.

It only remains to prove that (iii) implies $\lambda \in \sigma_{\text {ess }}(A, \mathscr{L})$. Each individual eigenvector of $A_{n_{k}}$ might not converge weakly to 0 , however there is a linear combination of them that does it. We prove this as follows. Let $\left(f_{j}^{k}\right)_{j=1}^{d+1}$ be an orthonormal set of $d+1$ eigenvectors

$$
A_{n_{k}} f_{j}^{k}=\lambda_{j}^{k} f_{j}^{k}, \quad j=1, \ldots, d+1 .
$$


Up to extraction of subsequences we may assume that $f_{j}^{k} \rightarrow f_{j} \in \operatorname{Ker}(A-\lambda)$ for all $j=1, \ldots, d+1$. If $f_{j}=0$ for some $j$, then the desired conclusion follows. Otherwise, since $\operatorname{dim} \operatorname{Ker}(A-\lambda)=d$, there exist coefficients $\left(a_{j}\right) \in \mathbb{C}^{d+1} \backslash\{0\}$ such that $\sum_{j=1}^{d+1} a_{j} f_{j}=0$. Therefore, we may take

$$
y_{k} \stackrel{\text { def }}{=} \frac{\sum_{j=1}^{d+1} a_{j} f_{j}^{k}}{\sqrt{\sum_{j=1}^{d+1}\left|a_{j}\right|^{2}}}
$$

as singular $\mathscr{L}$-Weyl sequence for $\lambda$. This completes the proof of Proposition 5.

\section{Mapping of the limiting spectra}

In this section we establish mapping theorems for the different limiting spectra. They are a natural generalisation of the analogous well-known result for $\sigma(A)$ and $\sigma_{\text {ess }}(A)$; see for example [29], Section XIII.4. From now on we assume that $A$ is bounded from below and we take $\mathscr{L}$ to be an $(A-a)^{1 / 2}$-regular Galerkin sequence with $a<\inf \sigma(A)$.

Theorem 7 (Mapping of the limiting spectra). Let $A$ be semi-bounded from below and let $a<\inf \sigma(A)$. Assume that $\mathscr{L}$ is an $(A-a)^{1 / 2}$-regular Galerkin sequence. Then

$$
\lambda \in \sigma(A, \mathscr{L}) \Longleftrightarrow(\lambda-a)^{-1} \in \sigma\left((A-a)^{-1}, \mathcal{E}\right)
$$

and

$$
\lambda \in \sigma_{\mathrm{ess}}(A, \mathscr{L}) \Longleftrightarrow(\lambda-a)^{-1} \in \sigma_{\mathrm{ess}}\left((A-a)^{-1}, \mathcal{E}\right),
$$

where $\mathscr{E}=\left((A-a)^{1 / 2} \mathscr{L}_{n}\right)_{n \in \mathbb{N}}$.

Remark 4. Recall that a selfadjoint operator $A$ is unbounded $(\mathrm{D}(A) \subsetneq \mathscr{H})$ if and only if $0 \in \sigma\left((A-a)^{-1}\right)$ for one (hence for all) $a \notin \sigma(A)$. As it turns out, $A$ is unbounded if and only if $0 \in \sigma_{\text {ess }}\left((A-a)^{-1}, \mathscr{E}\right)$ for one (and hence all) $a<\min \sigma(A)$ and $(A-a)^{1 / 2}$-regular sequence $\mathscr{L}$. Formally in Theorem 7 this corresponds to the case $+\infty \in \sigma(A)$ and $(+\infty-a)^{-1}=0$.

Evidently a result analogous to Theorem 7 can be established when $A$ is semibounded from above. However, here $A$ is required to be semi-bounded, in order to be able to use a square root $(A-a)^{1 / 2}$ in the definition of $\mathcal{E}$, and also for a more fundamental reason. When $a$ is in a gap of the essential spectrum, it would be natural to expect an extension of the above result by considering, for example, $\mathscr{E}=\left(|A-a|^{1 / 2} \mathscr{L}_{n}\right)_{n \in \mathbb{N}}$. The following shows that this extension is not possible in general. 
Example 5 (Impossibility of extending Theorem 7 for $A$ strongly indefinite). Let $\mathscr{H}$ be as in Example 2. Define $\mathscr{L}_{n}=\operatorname{Span}\left\{e_{1}^{ \pm}, \ldots, e_{n-1}^{ \pm}, \cos \left(\theta_{n}\right) e_{n}^{+}+\sin \left(\theta_{n}\right) e_{n}^{-}\right\}$ with $\theta_{n} \stackrel{\text { def }}{=} \pi / 4-\lambda /(2 n)$ for a fixed $\lambda \in(0,1)$. Let

$$
A=\sum n\left|e_{n}^{+}\right\rangle\left\langle e_{n}^{+}\left|-\sum n\right| e_{n}^{-}\right\rangle\left\langle e_{n}^{-}\right|
$$

Then $\sigma(A)=\{ \pm n: n \in \mathbb{N}\}=\sigma_{\text {disc }}(A)$. On the other hand

$$
\sigma(A, \mathscr{L})=\sigma(A) \cup\{\lambda\}, \quad \sigma_{\text {ess }}(A, \mathscr{L})=\{\lambda\} \quad \text { and } \quad \sigma_{\text {disc }}(A, \mathscr{L})=\sigma(A) .
$$

Now

$$
A^{-1}=\sum n^{-1}\left|e_{n}^{+}\right\rangle\left\langle e_{n}^{+}\left|-n^{-1}\right| e_{n}^{-}\right\rangle\left\langle e_{n}^{-}\right|
$$

and $\mathscr{E}=\sqrt{|A|} \mathscr{L}=\mathscr{L}$. Since $A^{-1}$ is compact we have

$$
\sigma\left(A^{-1}, \mathcal{G}\right)=\sigma\left(A^{-1}\right)
$$

and

$$
\sigma_{\mathrm{ess}}\left(A^{-1}, \mathcal{E}\right)=\sigma_{\mathrm{ess}}\left(A^{-1}\right)=\{0\}
$$

Thus $\lambda \in \sigma_{\text {ess }}(A, \mathscr{L})$ whereas $1 / \lambda \notin \sigma\left(A^{-1}, \mathscr{E}\right)$.

In fact the following example shows that no general extension of this theorem is possible whenever $a$ lies in the convex hull of the essential spectrum, even for $A \in \mathscr{B}(\mathscr{H})$.

Example 6 (Impossibility of extending Theorem 7 for $a \in \operatorname{Conv}\left\{\sigma_{\text {ess }}(A)\right\}$ ). Let $\mathscr{H}=L^{2}(-\pi, \pi)$ and $A f(x)=\operatorname{sgn}(x) f(x)$ for all $f \in \mathscr{H}$. Then $\sigma(A)=\sigma_{\text {ess }}(A)=$ $\{ \pm 1\}$. If $\mathscr{L}$ is any $A$-regular sequence, then $\sigma(A, \mathscr{L}) \subset[-1,1]$. Fixing $a=0$ yields $(A-a)^{-1}=A$. Thus also $\sigma\left(A^{-1}\right)=\{ \pm 1\}$ and $\sigma\left((A-a)^{-1}, \mathcal{G}\right) \subset[-1,1]$, for any $A$-regular sequence $\mathscr{G}$. If we construct a sequence $\mathscr{L}=\left(\mathscr{L}_{n}\right)$ leading to a spurious eigenvalue $\lambda \in \sigma_{\text {ess }}(A, \mathscr{L}) \cap(-1,1)$, we will always have $\lambda^{-1} \notin \sigma_{\text {ess }}\left(A^{-1}, \mathscr{E}\right)$, no matter what $\mathscr{E}$ is. We thus see that Theorem 7 cannot be extended to include $a$ in the convex hull of the essential spectrum.

Proof of Theorem 7. Statement (6) will follow immediately from the next result.

Lemma 8 (Mapping for the spectrum of compressions). Let $A$ be semi-bounded from below, let $a<\inf \sigma(A)$ and $\mathscr{L}_{n} \subset \mathrm{D}\left((A-a)^{1 / 2}\right)$. Then

$$
\lambda \in \sigma\left(\pi_{n} A \uparrow \mathscr{L}_{n}\right) \Longleftrightarrow(\lambda-a)^{-1} \in \sigma\left(p_{n}(A-a)^{-1} \uparrow \mathscr{E}_{n}\right)
$$

where $\mathscr{E}_{n}=(A-a)^{1 / 2} \mathscr{L}_{n}$ and $p_{n}$ is the associated orthogonal projector. 
Proof. Note that $\lambda \in \sigma\left(A_{n}\right)$ if and only if there exists $x \in \mathscr{L}_{n} \backslash\{0\}$ such that

$$
\pi_{n}(A-a)^{1 / 2}\left((\lambda-a)^{-1}-(A-a)^{-1}\right)(A-a)^{1 / 2} x=\frac{1}{\lambda-a} \pi_{n}(A-\lambda) x=0 .
$$

By fixing $y=(A-a)^{1 / 2} x \in \mathscr{E}_{n} \backslash\{0\}$, it is readily seen that $\lambda \in \sigma\left(A_{n}\right)$ if and only if there exist $y \in \mathscr{E}_{n} \backslash\{0\}$ such that

$$
\left\langle(A-a)^{1 / 2} u,\left((\lambda-a)^{-1}-(A-a)^{-1}\right) y\right\rangle=0
$$

for all $u \in \mathscr{L}_{n}$. Therefore, the statement $\lambda \in \sigma\left(A_{n}\right)$ is equivalent to the existence of $y \in \mathscr{G}_{n} \backslash\{0\}$ such that $\left((\lambda-a)^{-1}-(A-a)^{-1}\right) y \perp \mathscr{G}_{n}$ which, in turns, is equivalent to $p_{n}\left((\lambda-a)^{-1}-(A-a)^{-1}\right) y=0$.

We now turn to the proof of (7). We begin by establishing an alternative characterisation of the limiting essential spectrum and then we formulate a stability result for the limiting spectra with respect to compact perturbations of the regular Galerkin sequence.

Lemma 9 (Alternative characterisation of $\sigma_{\text {ess }}(A, \mathscr{L})$ ). Let

$$
\mathscr{F}(A) \stackrel{\text { def }}{=}\left\{f(A): f \in C_{c}\left(\mathbb{R} \backslash \sigma_{\text {ess }}(A), \mathbb{R}\right)\right\}
$$

and

$$
\mathcal{F}^{ \pm}(A) \stackrel{\text { def }}{=}\left\{f(A): f \in C_{c}\left(\mathbb{R} \backslash \sigma_{\mathrm{ess}}(A), \mathbb{R}^{ \pm}\right)\right\} .
$$

Then

$$
\begin{aligned}
\sigma_{\mathrm{ess}}(A, \mathscr{L}) & =\bigcap_{B \in \mathcal{F}(A)} \sigma(A+B, \mathscr{L}) \\
& =\bigcap_{B \in \mathcal{F}^{+}(A)} \sigma(A+B, \mathscr{L}) \\
& =\bigcap_{B \in \mathcal{F}^{-}(A)} \sigma(A+B, \mathscr{L}) .
\end{aligned}
$$

Here $C_{c}(\Omega, \mathbb{R})$ denotes the set of all real-valued continuous functions of compact support in the open set $\Omega$. Note that $\mathscr{F}(A)$ is a real vector space and $\mathscr{F}^{ \pm}(A)$ are cones, all spanned by projectors onto the eigenspaces of $A$ associated with isolated eigenvalues of finite multiplicity. At the end of this section it will become clear the reason why we highlight the right hand side characterisation in (8).

Proof of Lemma 9. We only prove the first equality of (8) as the proof of the other ones follows exactly the same pattern. It is well-known that

$$
\sigma_{\mathrm{ess}}(A)=\bigcap_{B \in \mathcal{F}(A)} \sigma(A+B) .
$$


Since all the operators in $\mathcal{F}(A)$ are of finite rank, then $\sigma_{\text {ess }}(A+B)=\sigma_{\text {ess }}(A)$ for all $B \in \mathscr{F}(A)$. Hence (9) is equivalent to

$$
\bigcap_{B \in \mathcal{F}(A)} \sigma_{\text {disc }}(A+B)=\varnothing .
$$

From Remark 2, it follows that $\sigma_{\text {ess }}(A+B, \mathscr{L})=\sigma_{\text {ess }}(A, \mathscr{L})$ for all $B \in \mathcal{F}(A)$. Therefore $\sigma(A+B, \mathscr{L})=\sigma_{\text {disc }}(A+B, \mathscr{L}) \cup \sigma_{\text {ess }}(A, \mathscr{L})$. Moreover $\sigma_{\text {disc }}(A+B, \mathscr{L}) \subseteq$ $\sigma_{\text {disc }}(A+B)$, by Proposition 3. Hence, by (10),

$$
\bigcap_{B \in \mathcal{F}(A)} \sigma_{\mathrm{disc}}(A+B, \mathscr{L}) \subset \bigcap_{B \in \mathcal{F}(A)} \sigma_{\mathrm{disc}}(A+B)=\varnothing
$$

and the result is proved.

Lemma 10. Let $T=T^{*}$ be such that $\|T\|<\infty$ and let $\mathscr{L}$ be a $T$-regular sequence. Let $K \in \mathcal{K}(\mathcal{H})$. If $-1 \notin \sigma(K)$, then

$$
\sigma_{\mathrm{ess}}(T, \mathscr{L})=\sigma_{\mathrm{ess}}(T,(1+K) \mathscr{L})
$$

and

$$
\sigma_{\text {disc }}(T, \mathscr{L})=\sigma_{\text {disc }}(T,(1+K) \mathscr{L})
$$

Proof of Lemma 10. We firstly prove that

$$
\sigma_{\mathrm{ess}}(T, \mathscr{L}) \backslash \sigma_{\mathrm{disc}}(T)=\sigma_{\mathrm{ess}}(T,(1+K) \mathscr{L}) \backslash \sigma_{\mathrm{disc}}(T) .
$$

Since

$$
\mathscr{L}=(1+K)^{-1}(1+K) \mathscr{L}=\left(1-K(1+K)^{-1}\right)(1+K) \mathscr{L},
$$

it suffices to show that the left hand side of (11) is contained in the right hand side. Let $\lambda \in \sigma_{\text {ess }}(T, \mathscr{L}) \backslash \sigma_{\text {disc }}(T)$. If $\lambda \in \sigma_{\text {ess }}(T)$, a direct application of Proposition 3(ii) ensures that $\lambda$ lies also in the right hand side of (11), so we can assume that $\lambda \notin$ $\sigma(A)$. According to Proposition 5(i), there exists $\lambda_{k} \rightarrow \lambda$ and $x_{k} \in \mathscr{L}_{n_{k}}$ such that $\left\|x_{k}\right\|=1, x_{k} \rightarrow 0$ and $\pi_{n_{k}}\left(T-\lambda_{k}\right) x_{k}=0$. For all $v_{k} \in \mathscr{L}_{n_{k}}$, and hence for all $w_{k}=(1+K) v_{k} \in(1+K) \mathscr{L}_{n_{k}}$, we have

$$
\begin{aligned}
0 & =\left\langle\left(T-\lambda_{k}\right) x_{k}, v_{k}\right\rangle \\
& =\left\langle\left(1+K^{*}\right)^{-1}\left(T-\lambda_{k}\right) x_{k},(1+K) v_{k}\right\rangle \\
& =\left\langle\left(1+K^{*}\right)^{-1}\left(T-\lambda_{k}\right) x_{k}, w_{k}\right\rangle .
\end{aligned}
$$

Let $q_{k}$ be the orthogonal projection onto $(1+K) \mathscr{L}_{n_{k}}$. Then

$$
q_{k}\left(1+K^{*}\right)^{-1}\left(T-\lambda_{k}\right) x_{k}=0 .
$$


Now $\left(1+K^{*}\right)^{-1}=1-\widetilde{K}$ where $\widetilde{K}=K^{*}\left(1+K^{*}\right)^{-1} \in \mathcal{K}(\mathscr{H})$. Hence

$$
q_{k}(1-\tilde{K})(T-\lambda) x_{k} \longrightarrow 0 .
$$

But, since $\|T\|<\infty$ and $x_{k} \rightarrow 0, \tilde{K}(T-\lambda) x_{k} \rightarrow 0$, so that also $q_{k}(T-\lambda) x_{k} \rightarrow 0$. Thus $q_{k}(T-\lambda) y_{k} \rightarrow 0$ for $y_{k}=(1+K) x_{k} \rightarrow 0$. By re-normalising $y_{k}$ in the obvious manner, we obtain a singular $\mathscr{L}$-Weyl sequence for $\lambda \in \sigma(T,(1+K) \mathscr{L})$, ensuring (11).

To complete the proof of the first identity in the conclusion of the lemma, suppose that $\lambda \in \sigma_{\text {ess }}(T, \mathscr{L}) \cap \sigma_{\text {disc }}(T)$. For any $\mu \neq \lambda$ let $\widetilde{T}=T+(\mu-\lambda) \mathbb{1}_{(\lambda-\varepsilon, \lambda+\varepsilon)}(T)$ where $\varepsilon>0$ is sufficiently small. Then $\lambda \in \sigma_{\text {ess }}(\widetilde{T}, \mathscr{L}) \backslash \sigma_{\text {disc }}(\widetilde{T})$. By virtue of (11) and Remark $2, \lambda \in \sigma_{\text {ess }}(\widetilde{T},(1+K) \mathscr{L})=\sigma_{\text {ess }}(T,(1+K) \mathscr{L})$ as needed.

We now show the second identity in the conclusion of the lemma. By virtue of (12) and the first identity which we just proved, it is enough to verify

$$
\sigma_{\text {disc }}(T, \mathscr{L}) \subset \sigma(T,(1+K) \mathscr{L}) .
$$

This, in turns, follows from Proposition 3-(ii) and (4), since

$$
\sigma_{\text {disc }}(T, \mathscr{L}) \subset \sigma_{\text {disc }}(T)
$$

and

$$
\sigma(T) \subset \sigma(T,(1+K) \mathscr{L})
$$

taking into account that $(1+K) \mathscr{L}$ is a $T$-regular sequence.

We now complete the proof of Theorem 7 by showing (7). Let $\lambda \in \sigma_{\text {ess }}(A, \mathscr{L})$. By virtue of Lemma 9, this is equivalent to the statement

$$
\lambda \in \sigma(A+B, \mathscr{L}) \text { for all } B \in \mathcal{F}^{+}(A) .
$$

Since $B \geq 0$ and $a<\min [\sigma(A+B)]$, according to (6) the latter is equivalent to

$$
(\lambda-a)^{-1} \in \sigma\left((A+B-a)^{-1}, \boldsymbol{E}_{B}\right) \text { for all } B \in \mathcal{F}^{+}(A)
$$

where $\mathscr{E}_{B}=(A+B-a)^{-1 / 2} \mathscr{L}$. Since $B$ has finite rank and is therefore compact, Lemma 10 ensures that the above in turns is equivalent to

$$
(\lambda-a)^{-1} \in \sigma\left((A+B-a)^{-1}, \mathscr{E}_{0}\right) \quad \text { for all } B \in \mathcal{F}^{+}(A) .
$$

Note that $0 \notin \sigma\left((A+B-a)^{1 / 2}(A-a)^{-1 / 2}\right)$ as the corresponding operator is an invertible function of $A$. Now $(A+B-a)^{-1}=(A-a)^{-1}+\widetilde{B}$, where $\widetilde{B}=$ $-(A-a)^{-1} B(A+B-a)^{-1}$ runs over all of $\mathscr{F}^{-}\left((A-a)^{-1}\right)$ as $B$ runs over all $\mathcal{F}^{+}(A)$ and conversely. For the latter note that $f \in \mathcal{F}^{+}(A)$ if and only if $-f\left((\cdot-a)^{-1}\right) \in$ $\mathcal{F}^{-}\left((A-a)^{-1}\right)$. Thus, once again by Lemma $9, \lambda \in \sigma_{\text {ess }}(A, \mathscr{L})$ is equivalent to

$$
(\lambda-a)^{-1} \in \sigma_{\mathrm{ess}}\left((A-a)^{-1}, \mathcal{E}\right) .
$$

This completes the proof of Theorem 7. 
Remark 5. The above proof mimics the proof of the classical Mapping Theorem for the essential spectrum which can be deduced from the characterisation

$$
\sigma_{\text {ess }}(A)=\bigcap_{B \in \mathcal{K}(\mathscr{H})} \sigma(A+B),
$$

see, e.g., [28].

\section{Stability properties of the limiting essential spectrum}

In this final section we present the main contribution of this paper. It strongly depends on the validity of Theorem 7 .

Theorem 11 (Weyl-type stability theorem for the limiting spectra). Let $A$ and $B$ be two selfadjoint operators which are bounded below. Assume that for some a $<$ $\inf \{\sigma(A), \sigma(B)\}$,

$$
\mathrm{D}\left((B-a)^{1 / 2}\right)=\mathrm{D}\left((A-a)^{1 / 2}\right)
$$

and

$$
(A-a)^{1 / 2}\left((B-a)^{-1 / 2}-(A-a)^{-1 / 2}\right) \in \mathcal{K}(\mathcal{H}) .
$$

Then

$$
\sigma_{\text {ess }}(A, \mathscr{L})=\sigma_{\text {ess }}(B, \mathscr{L})
$$

for all sequences $\mathscr{L}=\left(\mathscr{L}_{n}\right)$ which are simultaneously $(A-a)^{1 / 2}$-regular and $(B-a)^{1 / 2}$-regular.

Under assumption (13), condition (14) is equivalent to the same condition with the roles of $A$ and $B$ reversed:

$$
(B-a)^{1 / 2}\left((A-a)^{-1 / 2}-(B-a)^{-1 / 2}\right) \in \mathcal{K}(\mathcal{H}) .
$$

Note however that (13) and (14) do not imply necessarily that an $A$-regular sequence is also $B$-regular. For this it is enough to consider an example where $\mathrm{D}(A) \neq \mathrm{D}(B)$. Let $A=\partial_{x}^{4}$ with domain

$$
\mathrm{D}(A)=H^{4}(0,1) \cap\left\{u(0)=u(1)=0, u^{\prime \prime}(0)=u^{\prime \prime}(1)=0\right\} \subset L^{2}(0,1)=\mathscr{H} .
$$

Let $B=\partial_{x}^{4}+|1\rangle\langle 1|\left(1-\partial_{x}^{2}\right)$ with domain

$$
\mathrm{D}(B)=H^{4}(0,1) \cap\left\{u(0)=u(1)=0, u^{\prime \prime}(0)=u^{\prime \prime}(1)=\int_{0}^{1} u(x) \mathrm{d} x\right\} \neq \mathrm{D}(A) .
$$

Then $A^{1 / 2}=-\partial_{x}^{2}$ and $B^{1 / 2}=-\partial_{x}^{2}+|1\rangle\langle 1|$ both with domain

$$
\mathrm{D}\left(A^{1 / 2}\right)=\mathrm{D}\left(B^{1 / 2}\right)=H^{2}(0,1) \cap\{u(0)=u(1)=0\} .
$$

Since $A^{1 / 2}\left(B^{-1 / 2}-A^{-1 / 2}\right)=|1\rangle\langle 1| B^{-1 / 2}$ is a rank-one operator, $A$ and $B$ satisfy the hypotheses of Theorem 11, but clearly $A$-regular sequences $\mathscr{L}_{n} \subset \mathrm{D}(A) \backslash \mathrm{D}(B)$ are not $B$-regular. 
Remark 6. The KLMN theorem [29] ensures that if $B-A$ is a densely defined symmetric $A$-form-bounded operator with bound less than 1 , then (13) holds for $a$ sufficiently negative.

The following example from [22] shows that Theorem 11 cannot be easily generalised to operators which are not semi-bounded.

Example 7 (Relatively compact perturbations of the Dirac operator). Let $A=D^{0}$ and $B=D^{0}+V$ where $D^{0}$ denotes the free Dirac operator with unit mass [33] and $V \in C_{c}^{\infty}\left(\mathbb{R}^{3}\right)$ is a smooth non-negative function of compact support. The ambient Hilbert space here is $\mathscr{H}=L^{2}\left(\mathbb{R}^{3}, \mathbb{C}^{4}\right)$. Under the additional assumption that $\sup V=\|V\|_{L^{\infty}\left(\mathbb{R}^{3}\right)}<1$, it is guaranteed that $0 \notin \sigma(B)$. Furthermore it can be verified that

$$
D\left(|A|^{1 / 2}\right)=D\left(|B|^{1 / 2}\right)=H^{1 / 2}\left(\mathbb{R}^{3}, \mathbb{C}^{4}\right)
$$

and that

$$
|A|^{1 / 2}\left(|B|^{-1 / 2}-|A|^{-1 / 2}\right) \in \mathcal{K}(\mathscr{H}) .
$$

As a consequence of [22], Theorem 2.7, it is known that there exists a $B$-regular Galerkin sequence $\mathscr{L}=\left(\mathscr{L}_{n}\right)$ such that

$$
\sigma_{\text {ess }}(B, \mathscr{L}) \supset[0 ; \sup V] .
$$

These Galerkin spaces comprise upper and lower spinors, meaning that

$$
\mathscr{L}_{n}=\operatorname{Span}\left\{\left(\begin{array}{c}
f_{1}^{n} \\
0
\end{array}\right), \ldots,\left(\begin{array}{c}
f_{d_{n}}^{n} \\
0
\end{array}\right),\left(\begin{array}{c}
0 \\
g_{1}^{n}
\end{array}\right), \ldots,\left(\begin{array}{c}
0 \\
g_{d_{n}^{\prime}}^{n}
\end{array}\right)\right\}
$$

for suitable $\left(f_{j}^{n}\right),\left(g_{j}^{n}\right) \subset L^{2}\left(\mathbb{R}^{3}, \mathbb{C}^{2}\right)$. This basis is known to be free of pollution if the external field $V=0$, that is

$$
\sigma(A, \mathscr{L})=\sigma\left(D^{0}\right)=(-\infty,-1] \cup[1, \infty)=\sigma_{\text {ess }}(A, \mathscr{L}) .
$$

Hence $\sigma_{\text {ess }}(A, \mathscr{L}) \neq \sigma_{\text {ess }}(B, \mathscr{L})$ so Theorem 11 fails for operators which are strongly indefinite.

Proof of Theorem 11. Denote by $K$ the operator on the left side of (14). Then

$$
(B-a)^{-1}-(A-a)^{-1}=(A-a)^{-1 / 2} K(B-a)^{-1 / 2}+(A-a)^{-1} K \in \mathcal{K}(\mathscr{H}) .
$$

Let $\mathscr{\mathscr { d e f }} \stackrel{\text { d }}{=}(A-a)^{1 / 2} \mathscr{L}$. According to (7),

$$
\lambda \in \sigma_{\mathrm{ess}}(A, \mathscr{L}) \Longleftrightarrow(\lambda-a)^{-1} \in \sigma_{\mathrm{ess}}\left((A-a)^{-1}, \mathcal{E}\right) .
$$

By Remark 2,

$$
\sigma_{\mathrm{ess}}\left((B-a)^{-1}, \mathcal{E}\right)=\sigma_{\mathrm{ess}}\left((A-a)^{-1}, \mathcal{E}\right)
$$


Let $\mathscr{G}^{\prime}=(B-a)^{1 / 2} \mathscr{L}$. Then

$$
\mathcal{E}=(A-a)^{1 / 2} \mathscr{L}=(A-a)^{1 / 2}(B-a)^{-1 / 2} \mathscr{E}^{\prime}=(1+K) \mathscr{Y}^{\prime} .
$$

Note that $K=(A-a)^{1 / 2}(B-a)^{-1 / 2}-1$ and $-1 \notin \sigma(K)$ as a consequence of the fact that $0 \notin \sigma\left((A-a)^{1 / 2}(B-a)^{-1 / 2}\right)$ by (13). According to Lemma 10,

$$
\sigma_{\mathrm{ess}}\left((B-a)^{-1}, \mathcal{E}\right)=\sigma_{\mathrm{ess}}\left((B-a)^{-1}, \mathcal{E}^{\prime}\right) .
$$

The conclusion follows by applying Theorem 7 again, this time to operator $B$.

Corollary 12. Let $A$ and $B$ be two bounded-below selfadjoint operators such that (13) holds true for some $a<\inf \{\sigma(A), \sigma(B)\}$. Assume that $C \stackrel{\text { def }}{=} B-A$ is a densely defined symmetric operator such that

$$
C \in \mathscr{B}\left(\mathrm{D}\left((B-a)^{\beta}\right), \mathscr{H}\right)
$$

and

$$
(A-a)^{-\alpha} C(B-a)^{-\beta} \in \mathcal{K}(\mathscr{H})
$$

for some $0 \leq \alpha, \beta<1$ with $\alpha+\beta<1$. Then

$$
\sigma_{\mathrm{ess}}(A, \mathscr{L})=\sigma_{\mathrm{ess}}(B, \mathscr{L})
$$

for all sequences $\mathscr{L}=\left(\mathscr{L}_{n}\right)$ which are simultaneously $(A-a)^{1 / 2}$-regular and $(B-a)^{1 / 2}$-regular.

Remark 7. Let $A$ be a given bounded-below selfadjoint operator and assume that $A$ has a gap $(a, b)$ in its essential spectrum in the following precise sense,

$$
\sigma_{\mathrm{ess}}(A) \cap(a, b)=\varnothing, \quad \operatorname{tr}\left(\mathbb{1}_{(-\infty, a)}(A)\right)=\operatorname{tr}\left(\mathbb{1}_{(b, \infty)}(A)\right)=+\infty .
$$

Let $\Pi \stackrel{\text { def }}{=} \mathbb{1}_{(c, \infty)}(A)$ where $a<c<b$. Results shown in [22] ensure that, when the Galerkin spaces $\mathscr{L}_{n}$ are compatible with the decomposition $\mathscr{H}=\Pi \mathscr{H} \oplus(1-\Pi) \mathscr{H}$ (i.e. when $\Pi$ and $\pi_{n}$ commute for all $n$ ), there is no pollution in the gap: $\sigma_{\text {ess }}(A, \mathscr{L}) \cap$ $(a, b)=\varnothing$. According to [22], Corollary 2.5, when

$$
(B-a)^{-1} C(A-a)^{-1 / 2} \in \mathcal{K}(\mathscr{H}),
$$

then $\sigma_{\text {ess }}(B, \mathscr{L})=\varnothing$ as well.

In this respect, Theorem 11 can be seen as a generalisation of these results. Although condition (20) is stronger than (21), the statement guarantees that the whole polluted spectrum will not move irrespectively of the $(A-a)^{1 / 2}$-regular Galerkin family $\mathscr{L}$ and not only for those satisfying $\left[\Pi, \pi_{n}\right]=0$ for all $n$. 
Example 8 (Periodic Schrödinger operators). Let $A=-\Delta+V_{\text {per }}$ where $V_{\text {per }}$ is a periodic potential with respect to some fixed lattice $\mathcal{R} \subset \mathbb{R}^{d}$ (for instance $\mathcal{R}=\mathbb{Z}^{d}$ ). Let $C=W(x)$ be a perturbation. Assume that

$$
V_{\text {per }} \in L_{\mathrm{loc}}^{p}\left(\mathbb{R}^{d}\right) \quad \text { where } \begin{cases}p=2 & \text { if } d \leq 3, \\ p>2 & \text { if } d=4, \\ p=d / 2 & \text { if } d \geq 5,\end{cases}
$$

and that

$$
W \in L^{q}\left(\mathbb{R}^{d}\right) \cap L_{\mathrm{loc}}^{p}\left(\mathbb{R}^{d}\right)+L_{\varepsilon}^{\infty}\left(\mathbb{R}^{d}\right)
$$

for $\max (d / 2,1)<q<\infty$. Then (20) holds true for suitable $\alpha, \beta$ and $a$, and therefore

$$
\sigma_{\text {ess }}\left(-\Delta+V_{\text {per }}+W, \mathscr{L}\right)=\sigma_{\text {ess }}\left(-\Delta+V_{\text {per }}, \mathscr{L}\right)
$$

for all $A$-regular Galerkin sequence $\mathscr{L}$. See [22], Section 2.3.1.

A Galerkin sequence $\mathscr{L}$ which does lead to any pollution in a given gap, can be found by localised Wannier functions; see [22] and [9]. In practice, these functions can only be calculated numerically, so it is natural to ask what would be the polluted spectrum when they are known only approximately. According to (22), the polluted spectrum will not increase in size more than that of the unperturbed operator $-\Delta+V_{\text {per }}$.

Example 9 (Optimality of the constants in Corollary 12). Let $\mathscr{H}, \mathscr{L}, e_{n}^{ \pm}$and $f_{n}^{ \pm}$be as in Example 2. Let

$$
A=\sum_{n} n^{\ell}\left|f_{n}^{+}\right\rangle\left\langle f_{n}^{+}\left|+\sum\right| f_{n}^{-}\right\rangle\left\langle f_{n}^{-}\right|
$$

and

$$
B=\sum_{n} n^{r}\left|e_{n}^{+}\right\rangle\left\langle e_{n}^{+}\left|+\sum\right| e_{n}^{-}\right\rangle\left\langle e_{n}^{-}\right|
$$

The matrix representation of $A$ and $B$ in the basis $e_{n}^{ \pm}$is made out of $2 \times 2$ blocks placed along the diagonal. More precisely $A=\operatorname{diag}\left[A_{n}\right], B=\operatorname{diag}\left[B_{n}\right]$ and $C=\operatorname{diag}\left[C_{n}\right]$; where

$$
\begin{aligned}
& A_{n}=R_{-n}\left(\begin{array}{cc}
n^{\ell} & 0 \\
0 & 1
\end{array}\right) R_{n}, \\
& B_{n}=\left(\begin{array}{cc}
n^{r} & 0 \\
0 & 1
\end{array}\right)
\end{aligned}
$$

and

$$
C_{n}=A_{n}-B_{n}
$$


for

$$
R_{n}=\left(\begin{array}{cc}
\cos \frac{1}{n} & \sin \frac{1}{n} \\
-\sin \frac{1}{n} & \cos \frac{1}{n}
\end{array}\right) .
$$

Fix $a=0$ and let $L=A^{-\alpha} C B^{-\beta}$. The matrix representation of $L$ in the basis $e_{k}^{ \pm}$is $L=\operatorname{diag}\left[L_{n}\right]$ where we can calculate explicitly the entries as

$$
\begin{aligned}
\left(L_{n}\right)_{11}= & -n^{-\beta r-\alpha \ell+r} \cos ^{2} \frac{1}{n}+n^{-\beta r} \sin ^{2} \frac{1}{n} \\
& -n^{-r(\beta-1)} \sin ^{2} \frac{1}{n}+n^{-\beta r-\alpha \ell+\ell} \cos ^{2} \frac{1}{n}, \\
\left(L_{n}\right)_{12}= & \cos \frac{1}{n} \sin \frac{1}{n}\left(n^{-\ell(\alpha-1)}-n^{-\alpha \ell}\right), \\
\left(L_{n}\right)_{21}= & \cos \frac{1}{n} \sin \frac{1}{n}\left(n^{-\beta r-\alpha \ell+\ell}-n^{-\beta r-\alpha \ell+r}-n^{-\beta r}+n^{-r(\beta-1)}\right), \\
\left(L_{n}\right)_{22}= & \sin ^{2} \frac{1}{n}\left(n^{-\ell(\alpha-1)}-n^{-\alpha \ell}\right) .
\end{aligned}
$$

Therefore $L$ is compact, given the following

$$
\begin{gathered}
\ell=2, \quad 0<\beta, \alpha<1, \quad 0<r<2, \\
-\beta r-2 \alpha+2<0, \quad \alpha>1 / 2, \quad \beta>1-\frac{1}{r} .
\end{gathered}
$$

On the other hand, for $\ell=2$,

$$
\sigma_{\text {ess }}(A, \mathscr{L})=\{1,2\} \quad \text { and } \quad \sigma_{\text {ess }}(B, \mathscr{L})=\{1\} .
$$

This example suggests that condition (20) in Corollary 12 is quasi-optimal for the stated range of $\beta$ and $\alpha$ as illustrated by Figure 1. Note however that in this example (13) is only satisfied when $r=\ell$.

Proof of Corollary 12. Assume firstly that $0 \leq \alpha \leq 1 / 2$. The proof reduces to showing that the operator $K$ defined by expression (14) is compact. Let

$$
L=(A-a)^{-\alpha} C(B-a)^{-\beta}
$$

be the operator given by (20). Since $\beta<1$, we have $\mathrm{D}(B-a) \subset \mathrm{D}(B-a)^{\beta}$; see [11], Theorem 4.3.4. Then, by (19), $L \mathscr{H} \subset \mathrm{D}\left((A-a)^{\alpha}\right)$ and $C x=(A-a)^{\alpha} L(B-a)^{\beta} x$ for all $x \in \mathrm{D}(B-a)$. By virtue of (20),

$$
(A-a)^{1 / 2}(A-a+s)^{-1} C(B-a+s)^{-1} \in \mathcal{K}(\mathscr{H})
$$




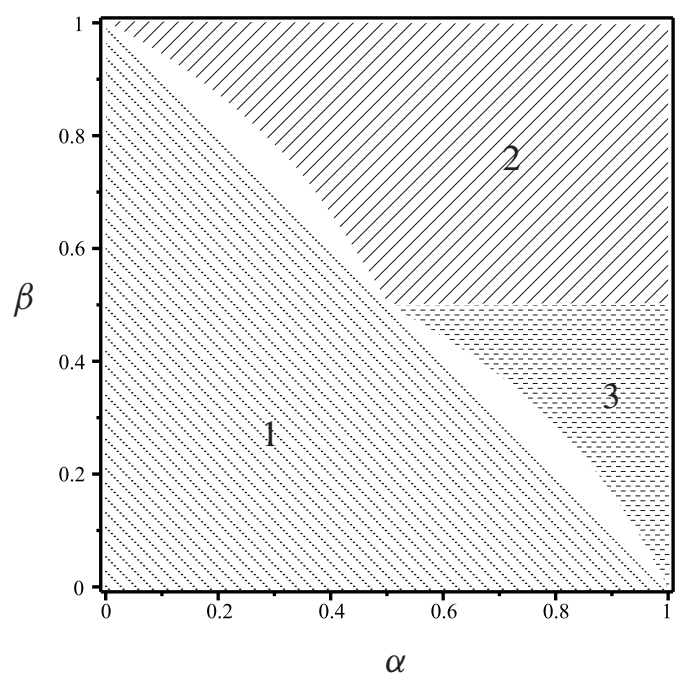

Figure 1. The region labelled 1 for the parameters $\beta$ and $\alpha$ is covered by the conditions of Corollary 12. If $A$ and $B$ satisfy (20) for $(\beta, \alpha)$ in this region, then the limiting essential spectrum is preserved. The region labelled 2 shows the parameters $\beta$ and $\alpha$ in condition (23) of Example 9. The region labelled 3 is generated by exchanging the roles of $\beta$ and $\alpha$. It is not enough for $A$ and $B$ to satisfy (20) for $(\beta, \alpha)$ in these two regions, to guarantee preservation of the limiting essential spectrum.

for all $s \geq 0$. Moreover

$$
\begin{aligned}
& (A-a)^{1 / 2}\left((A-a+s)^{-1}-(B-a+s)^{-1}\right) x \\
& \quad=(A-a)^{1 / 2}(A-a+s)^{-1} C(B-a+s)^{-1} x
\end{aligned}
$$

for all $x \in \mathscr{H}$, as this identity is satisfied in a dense subspace of $\mathscr{H}$. Thus

$$
\begin{array}{r}
K=-\frac{1}{\pi} \int_{0}^{\infty}(A-a)^{1 / 2}(A-a+s)^{-1} C(B-a+s)^{-1} \frac{\mathrm{d} s}{\sqrt{s}} \\
=-\frac{1}{\pi} \int_{0}^{\infty}\left\{(A-a)^{1 / 2}(A-a+s)^{-1}(A-a)^{\alpha}\right\} \\
L\left\{(B-a)^{\beta}(B-a+s)^{-1}\right\} \frac{\mathrm{d} s}{\sqrt{s}} .
\end{array}
$$

Both terms in brackets multiplying $L$ are bounded operators, then the integrand in the second expression is also a compact operator. Moreover, the integral converges in the Bochner sense as its norm is $O\left(s^{\beta+\alpha-2}\right)$ for $s \rightarrow \infty$ and $O\left(s^{-1 / 2}\right)$ for $s \rightarrow 0$. Thus $K \in \mathcal{K}(\mathscr{H})$ in this case and Theorem 11 implies the desired conclusion.

Now suppose that $1 / 2<\alpha<1$, so that $0 \leq \beta \leq 1 / 2$. Since

$$
\mathrm{D}(A-a)^{\alpha} \subset \mathrm{D}(A-a)^{1 / 2}=\mathrm{D}(B-a)^{1 / 2} \subset \mathrm{D}(B-a)^{\beta},
$$


then $C \in \mathscr{B}\left(\mathrm{D}(A-a)^{\alpha}, \mathscr{H}\right)$. Hence the operator $(B-a)^{-\beta} C(A-a)^{-\alpha}$ is bounded and $(B-a)^{-\beta} C(A-a)^{-\alpha} x=L^{*} x$ for all $x \in \mathscr{H}$. The proof is then completed by exchanging the roles of $A$ and $B$.

\section{References}

[1] L. Aceto, P. Ghelardoni, and M. Marletta, Numerical computation of eigenvalues in spectral gaps of Sturm-Liouville operators. J. Comput. Appl. Math. 189 (2006), 453-470. MR 2202990 Zbl 1104.65080

[2] D. Arnold, R. S. Falk, and R. Winther, Finite element exterior calculus: from Hodge theory to numerical stability. Bull. Amer. Math. Soc. 47 (2010), 281-354. MR 2594630 Zbl 1207.65134

[3] F. V. Atkinson, H. Langer, R. Mennicken, and A. A. Shkalikov. The essential spectrum of some matrix operators. Math. Nachr. 167 (1994), 5-20. MR 1285306 Zbl 0831.47001

[4] D. Boffi, F. Brezzi, and L. Gastaldi, Mixed finite elements for Maxwell's eigenproblem: the question of spurious modes. In H. G. Bock et al. (eds.), ENUMATH 97. Proceedings of the Second European conference on numerical mathematics and advanced applications held in Heidelberg, Germany, September 28-October 3, 1997. Including a selection of papers from the 1st conference (ENUMATH 95) held in Paris, France, September 1995. World Scientific, Singapore etc., 1998, 180-187. MR 1705031 Zbl 0992.78509

[5] D. Boffi, F. Brezzi, and L. Gastaldi, On the problem of spurious eigenvalues in the approximation of linear elliptic problems in mixed form. Math. Comp. 69 (2000). MR 1642801 Zbl 0938.65126

[6] L. Boulton and N. Boussaid, Non-variational computation of the eigenstates of Dirac operators with radially symmetric potentials. LMS J. Comput. Math. 13 (2010), 10-32. MR 2593910 Zbl 1227.65105

[7] L. Boulton and M. Levitin, On approximation of the eigenvalues of perturbed periodic Schrödinger operators. J. Phys. A 40 (2007), 9319-9329. MR 2345295 Zbl 1120.81033

[8] L. Boulton and M. Strauss, On the convergence of second order spectra and multiplicity. Proc. R. Soc. Lond., Ser. A, Math. Phys. Eng. Sci. 467 (2010), 264-284. MR 2764683 Zbl 1219.65051

[9] É. Cancès, A. Deleurence, and M. Lewin, Non-perturbative embedding of local defects in crystalline materials. J. Phys.: Condens. Matter 20 (2008), 294213.

[10] M. Dauge and M. Suri, Numerical approximation of the spectra of non-compact operators arising in buckling problems. J. Numer. Math. 10 (2002), 193-219. MR 1935966 Zbl 1099.74545

[11] E. B. Davies, Spectral theory and differential operators. Cambridge Studies in Advanced Mathematics 42. Cambridge University Press, Cambridge, 1995. MR 1349825 Zbl 0893.47004

[12] E. B. Davies and M. Plum, Spectral pollution. IMA J. Numer. Anal. 24 (2004), 417-438. MR 2068830 Zbl 1062.65056 
[13] J. Descloux, Essential numerical range of an operator with respect to a coercive form and the approximation of its spectrum by the Galerkin method. SIAM J. Numer. Anal. 18 (1981), 1128-1133. MR 0639003 Zbl 0523.65040

[14] J. Dolbeault, M. J. Esteban, and E. Séré, On the eigenvalues of operators with gaps. Application to Dirac operators. J. Funct. Anal. 174 (2000), 208-226. MR 1761368 Zbl 0982.47006

[15] G. W. F. Drake and S. P. Goldman, Application of discrete-basis-set methods to the Dirac equation. Phys. Rev. A 23 (1981), 2093-2098.

[16] M. J. Esteban, M. Lewin, and E. Séré, Variational methods in relativistic quantum mechanics. Bull. Am. Math. Soc., New Ser. 45 (2008), 535-593. MR 2434346Zbl 05348713

[17] I. P. Grant, Conditions for convergence of variational solutions of Dirac's equation in a finite basis. Phys. Rev. A 25 (1982), 1230-1232.

[18] A. C. Hansen, On the approximation of spectra of linear operators on Hilbert spaces. J. Funct. Anal. 254 (2008), 2092-2126. MR 2402104 Zbl 1138.47002

[19] M. Kraus, M. Langer, and C. Tretter, Variational principles and eigenvalue estimates for unbounded block operator matrices and applications. J. Comput. Appl. Math. 171 (2004), 311-334. MR 2077211 Zbl 1066.49030

[20] W. Kutzelnigg, Basis set expansion of the Dirac operator without variational collapse. Int. J. Quantum Chemistry 25 (1984), 107-129.

[21] M. Levitin and E. Shargorodsky, Spectral pollution and second-order relative spectra for self-adjoint operators. IMA J. Numer. Anal. 24 (2004), 393-416. MR 2068829 Zbl 1060.65056

[22] M. Lewin and É. Séré, Spectral pollution and how to avoid it (with applications to Dirac and periodic Schrödinger operators). Proc. Lond. Math. Soc. (3) 100 (2010), 864-900. MR 2640293 Zbl 1192.47016

[23] M. Marletta, Neumann-Dirichlet maps and analysis of spectral pollution for non-selfadjoint elliptic PDEs with real essential spectrum. IMA J. Numer. Anal. 30 (2010), 887-897. MR 2727810 Zbl 1206.65245

[24] U. Mertins, On the convergence of the Goerisch method for self-adjoint eigenvalue problems with arbitrary spectrum. Z. Anal. Anwendungen 15 (1996), 661-686. MR 1406082 Zbl 0898.49020

[25] A. Pokrzywa, Method of orthogonal projections and approximation of the spectrum of a bounded operator. Studia Math. 65 (1979), 21-29. MR 0554538 Zbl 0437.47004

[26] A. Pokrzywa, Method of orthogonal projections and approximation of the spectrum of a bounded operator II. Studia Math. 70 (1981), 1-9. MR 0646956 Zbl 0509.47010

[27] J. Rappaz, J. Sanchez Hubert, E. Sanchez Palencia, and D. Vassiliev, On spectral pollution in the finite element approximation of thin elastic "membrane" shells. Numer. Math. $\mathbf{7 5}$ (1997), 473-500. MR 1431212 Zbl 0874.73066

[28] M. Reed and B. Simon, Methods of Modern Mathematical Physics I. Functionnal Analysis. Academic Press, New York etc., 1972. MR 0493419 Zbl 0242.46001

[29] M. Reed and B. Simon, Methods of modern mathematical physics IV. Analysis of operators. Academic Press, New York etc., 1978. MR 0493420 Zbl 0308.47002 
[30] R. E. Stanton and S. Havriliak, Kinetic balance: A partial solution to the problem of variational safety in Dirac calculations. J. Chem. Phys. 81 (1984), 1910-1918.

[31] G. Stolz and J. Weidmann, Approximation of isolated eigenvalues of ordinary differential operators. J. Reine Angew. Math. 445 (1993), 31-44. MR 1244968 Zbl 0781.34052

[32] G. Stolz and J. Weidmann, Approximation of isolated eigenvalues of general singular ordinary differential operators. Results Math., 28 (1995), 345-358. MR 1356897 Zbl 0842.47029

[33] B. Thaller, The Dirac equation. Texts and Monographs in Physics. Springer Verlag, Berlin, 1992. MR 1219537 Zbl 0765.47023

Received June 24, 2011; revised November 25, 2011

Lyonell Boulton, Department of Mathematics and Maxwell Institute for Mathematical Sciences, Heriot-Watt University, Edinburgh EH14 4AS, United Kingdom

E-mail: L.Boulton@hw.ac.uk

Nabile Boussaïd, Département de Mathématiques (CNRS-UMR 6623),

UFR Sciences et techniques, 16 route de Gray, 25030 Besançon cedex, France

E-mail: nabile.boussaid@univ-fcomte.fr

Mathieu Lewin, CNRS \& Département de Mathématiques (CNRS-UMR 8088),

Université de Cergy-Pontoise, 95000 Cergy-Pontoise, France

E-mail: Mathieu.Lewin@math.cnrs.fr 